\title{
Trapping Sites of Polypropylene Mechano-Radicals
}

\author{
Norio Kurokawa, Masato Sakaguchi, ${ }^{*}$ and Junkichi Sohma \\ Faculty of Engineering, Hokkaido University, Sapporo 060, Japan.
}

(Received July 19, 1977)

\begin{abstract}
The behaviors of free radicals produced by ball-milling of solid polypropylene (PP mechano-radicals) were studied by ESR and compared with those of radicals produced by $\gamma$-irradiation ( $\gamma$-radicals). The mechano-radicals were found to react more efficiently than the $\gamma$-radicals with oxygen as well as with the bulky molecule, methyl methacrylate. The peroxy mechano-radicals, which were formed by reaction of the mechano-radicals with oxygen, were very mobile and decayed more readily than the peroxy $\gamma$-radicals. The photochemical reaction of the peroxy mechano-radicals was studied, and the radicals produced by illumination of the peroxy mechano-radicals returned to the peroxy radical at $10^{-4}$ Torr by consuming the sorbed oxygen. From these experimental results the conclusion is drawn that the PP mechano-radicals were produced and trapped on the fresh surfaces created by fractures of solid polypropylene.

KEY WORDS Mechanical Fracture / Mechano-Radicals / ESR / Polypropylene /
\end{abstract}

It has been established that free radicals are produced at low temperatures by mechanical fracture, such as ball-milling or sawing. ${ }^{1-9}$ A radical mechanically produced is called a "Mechano-radical." The formation of mechanoradicals is always accompanied by the creation of fresh surfaces by ruptures of the sample, and therefore it is reasonably assumed that mechanoradicals are formed on the fresh surfaces. However, this assumption does not directly mean that the mechano-radicals, from which one can observe ESR spectra, are trapped on the fresh surfaces produced mechanically, because a radical in a polymer matrix can migrate from one site to another even at low temperatures. ${ }^{10-12}$ Accordingly, one can not rule out the possibility that the mechano-radicals formed on the fresh surfaces migrate into the polymer matrix before ESR observation. Experimental evidence is needed to determine whether or not mechanoradicals are trapped on the fresh surfaces. The purpose of this paper is to provide both the

\footnotetext{
* Present Address: Ichimura College, Inuyama 484,
} Japan. experimental evidence and the discussion which convince us that the trapping of the mechanoradicals occurs on the fresh surfaces. Polypropylene was chosen as the sample on which the data were collected, for polypropylene radicals produced either by irradiation ${ }^{13-16}$ or mechanically ${ }^{8,9,17}$ have been sufficiently studied by ESR. A radical produced by $\gamma$-irradiation to a sample is abbreviated as a " $\gamma$-Radical" in this paper.

\section{EXPERIMENTAL}

\section{Samples}

Isotactic polypropylene (PP) manufactured by Mitsui Petrochemical Co. was used for the samples. The methyl methacrylate (MMA) used in the scavenging experiment was supplied by the Wako Chemical Co.

\section{Production of Mechano-Radicals}

Mechano-radicals were produced by the ballmill method described in detail in a previous paper. ${ }^{8}$ Mechanical fractures were done at $77 \mathrm{~K}$ in the reduced pressure of $10^{-4}$ Torr. The reac- 
tion with MMA was carried out by using a special ampoule ${ }^{9}$ designed for reactions with mechano-radicals. Peroxy mechano-radicals were directly formed at $77 \mathrm{~K}$, except for the experiments on the reactivity with oxygen, by the ball-milling of samples in the presence of oxygen at 20 Torr.

\section{ESR Measurement}

ESR spectra were obtained on a PE- $1 \mathrm{X}$ band spectrometer (JEOL) with $100-\mathrm{kHz}$ modulation. Observations at $199 \mathrm{~K}$ were carried out by using a mixture of Dry-Ice and $n$-hexane as a coolant in the insert Dewar.

\section{RESULTS}

\section{Reactivity with Oxygen}

PP radicals, which had been formed either mechanically or by $\gamma$-irradiation in vacuum, were brought into contact with air at $77 \mathrm{~K}$, and the sample was heat-treated in air for five minutes at various temperatures. Changes in the ESR line-shape were found after the heattreatment, and this change was due to the mixing of the spectrum of the peroxy radicals. ${ }^{17,18}$ The fraction of the peroxy radical was quantitatively estimated from the double integrations of the peroxy radical component in the decomposition of the observed spectrum. In this estimation the percentage was taken to the total intensity of the ESR spectrum observed initially at $77 \mathrm{~K}$. The percentage is plotted against the temperature of heat-treatment in Figure 1. Complete conversion to the peroxy radicals was obtained for the mechano-radical at nearly $200 \mathrm{~K}$, at which

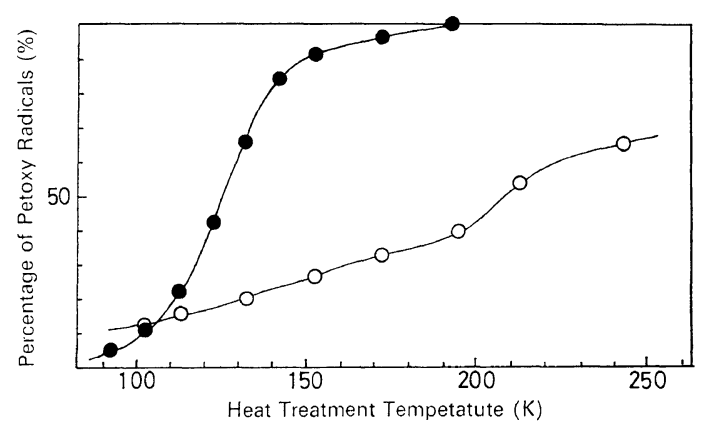

Figure 1. Reactivity of the PP radicals: mechano-radicals; $\bigcirc, \gamma$-radical; ESR observed at $77 \mathrm{~K}$. less than fifty percent of the $\gamma$-radicals were changed into peroxy radicals. This comparison clearly demonstrates the high reactivity of the mechano-radicals formed by milling with oxygen.

\section{Decay of the Peroxy Radicals}

Isothermal decays of the peroxy mechanoradicals as well as the peroxy $\gamma$-radicals were traced and are shown in Figure 2. There is a clear difference in the decay behavior of the peroxy radicals which have different origins. Approximately $90 \%$ of the peroxy mechanoradicals decay very rapidly, while the fraction of the radicals having a similar decay rate is only $50 \%$ in the case of the $\gamma$-radicals. Since fast decay is attributed to the peroxy radicals trapped in the amorphous region of the solid polypropylene,${ }^{19}$ the experimental result indicates that $c a .90 \%$ of the mechano-radicals are trapped in the amorphous region. ESR spectra observed at $199 \mathrm{~K}$ from the peroxy mechano-radicals are shown in Figure 3. " $A$ " and " $C$ " are the spectra of the peroxy mechano-radicals and of the peroxy $\gamma$-radicals, respectively. There is clearly a difference in the line-shape. The characteristic asymmetry of the pattern " $C$ " demonstrates that the responsible peroxy radicals are still frozen at this temperature, while the nearly symmetric shape of the spectrum " $A$ " means that the peroxy mechano-radicals are sufficiently mobile to undergo a partial averaging ${ }^{20}$ of the

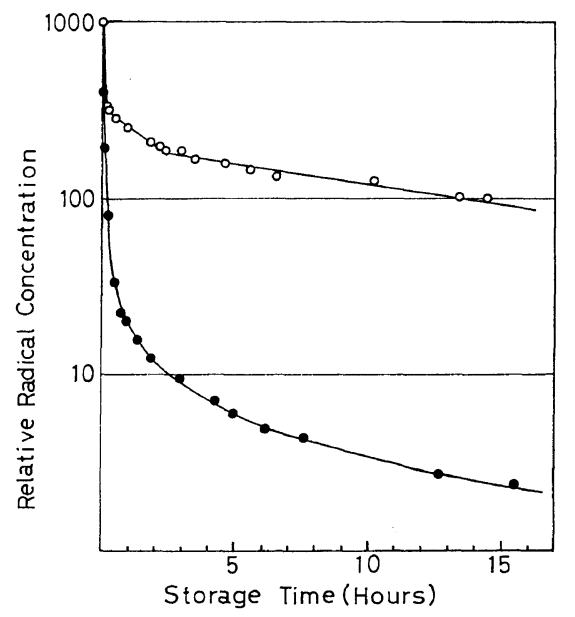

Figure 2. Isothermal decay of the peroxy radicals at $313 \mathrm{~K}$ : mechano-radical; $\bigcirc, \gamma$-radical; ESR observed at $77 \mathrm{~K}$. 


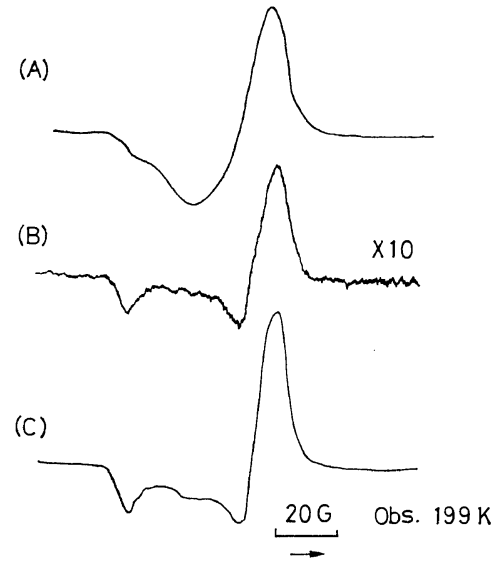

Figure 3. ESR line shape observed at 199K: (A) peroxy mechano-radical before the heat-treatment; (B) peroxy mechano-radical after the heat-treatment at $317 \mathrm{~K}$ (Gain is increased ten times.); (C) Peroxy $\gamma$-radical.

anisotropic $g$ factor. ${ }^{18}$ After the heat-treatment at $317 \mathrm{~K}$ the ESR spectrum observed at $199 \mathrm{~K}$ is shown as " $B$ " in the same figure. Although the spectrum was found to be weakened very much, the line-shape of the surviving peroxy radical appears asymmetric. From these experimental results one may conclude that the majority of the peroxy mechano-radicals are trapped in a region in which both rapid molecular motion and easy decay are permitted.

Reactivity with Methyl Methacrylate

PP mechano-radicals were brought into contact with methyl-methacrylate (MMA) at $77 \mathrm{~K}$ by making use of the special ampoule. In Figure 4

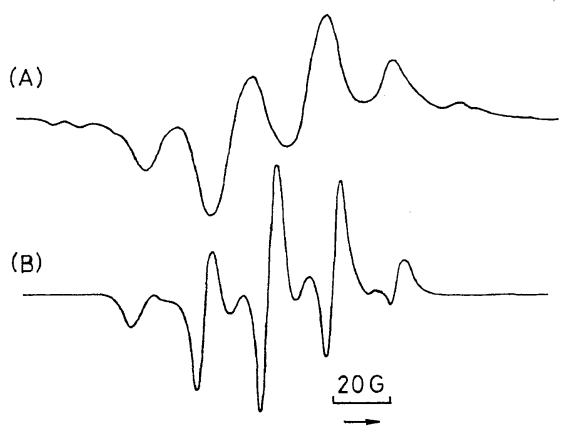

Figure 4. Comparison of the ESR line shapes observed from PP mechano-radicals in contact with MMA monomers: (A) before and (B) after the heat-treatment; ESR; observed at $77 \mathrm{~K}$.

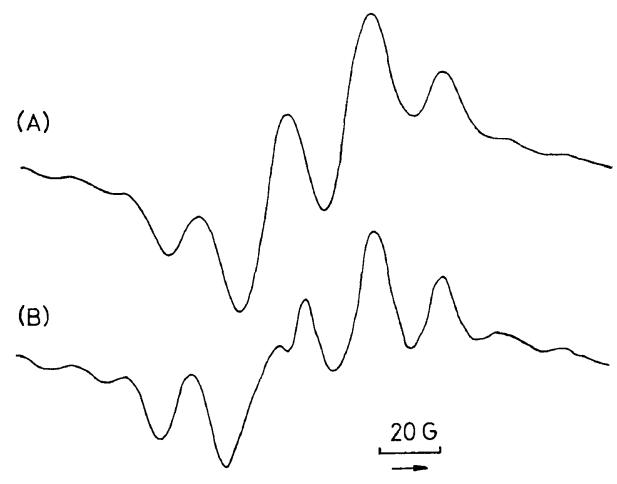

Figure 5. Comparison of the ESR line shapes observed from PP $\gamma$-radicals in contact with MMA monomers: (A) before and (B) after the heat treatment; ESR observed at $77 \mathrm{~K}$.

the ESR spectra observed before and after the heat-treatment at $273 \mathrm{~K}$ for five minutes are shown. " $A$ " is the spectrum observed from the fractured PP immediately after the contact with MMA monomer at $77 \mathrm{~K}$ and " $\mathrm{B}$ " is the lineshape observed from the same sample after the heat-treatment. The line-shape " $A$ " is the spectrum of PP mechano-radicals, ${ }^{17}$ while " $\mathrm{B}$ " is almost identical to the characteristic spectrum of the PMMA propagating radical, ${ }^{21}$ though a small component from the PP mechano-radicals is involved. These facts demonstrate that the PP mechano-radicals were almost completely scavenged by MMA monomers by the short heat-treatment at $0^{\circ} \mathrm{C}$. To make a comparion with the mechano-radicals behavior a similar experiment was carried out for the PP $\gamma$-radicals. The spectra before and after the heat-treatment are presented in Figure 5. Apparently no drastic change was caused by the heat-treatment of the $\gamma$-irradiated PP with MMA monomers.

Photo conversion of the Peroxy Mechano-Radicals

The PP mechano-radicals are easily converted into the peroxy mechano-radicals through contact with air, as mentioned above. After the complete conversion to the peroxy mechano-radicals the sample tube was evacuated to $10^{-4}$ Torr at $77 \mathrm{~K}$ and sealed off. It was experimentally confirmed by ESR observation that the peroxy radicals were survived by this evacuation. The peroxy mechano-radicals trapped in the fractured $\mathrm{PP}$ in the evacuated tube were irradiated at $77 \mathrm{~K}$ by UV light from a high pressure mercury lamp 


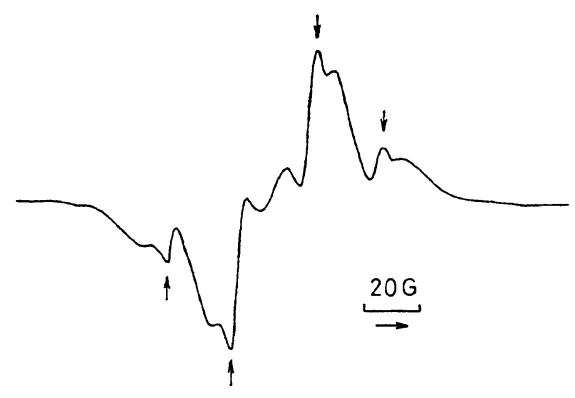

Figure 6. ESR spectrum observed after UV irradiation of the peroxy mechano-radicals; ESR observed at 77K. Arrows indicate the methyl quartet.

(Toshiba HP 400). The ESR spectrum resulting from the UV irradiation of the peroxy mechanoradicals is shown in Figure 6. This spectrum is markedly different from the spectrum of the peroxy radicals. A clear quartet, indicated by the arrows, is assigned to methyl radicals; the main component of the spectrum is not firmly identified yet, but presumably is $\sim$ C $(\mathbf{R} \cdot){ }^{15}$ The temperature of the sample was raised to $199 \mathrm{~K}$ and after this treatment ESR spectra were observed at $77 \mathrm{~K}$. It was surprising to find that the radicals produced by UV irradiation returned to the peroxy radicals because of the heat-treatment of the sample in the evacuated tube. These results are summarized as follows:

$$
\begin{aligned}
& \text { ROO. (at } 10^{-4} \text { Torr) } \\
& \underset{h_{\nu}}{\stackrel{\text { Thermal }}{\longrightarrow}} \cdot \mathrm{CH}_{3}+\mathrm{R} \cdot\left(\text { at } 10^{-4} \text { Torr }\right)
\end{aligned}
$$

This irradiation and heat-treatment procedure was repeated for the same sample. The same reversible reaction was observed in each repetition, but the relative yields of the peroxy radicals were found to decrease abruptly with the number of repetitions, as shown in Figure 7. The sharp decrease in this plot strongly suggests that the apparent reversible reaction (1) is not genuinely reversible but is a reaction which consumes some stored oxygen in the evacuated tube. The conversion of R. to ROO. after contact with oxygen gas at $10^{-4}$ Torr was very slow. Hence this rapid return to ROO. is

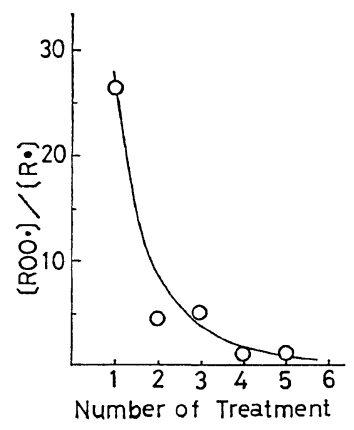

Figure 7. Relative yields of peroxy radicals by the repeated heat-treatments of the sample which gave the spectrum in Figure 6; ESR observed at $77 \mathrm{~K}$.

presumably due to a reaction not with oxygen gas in the tube but with oxygen adsorbed at $77 \mathrm{~K}$. In order to clarify the adsorption sites of the oxygen, either wall of the sample tube or surface of the sample polymer, similar experiments were carried out for the particular sample described below. After all of the peroxy mechano-radicals were annihilated by annealing at a elevated temperature, a sample tube containing this sample was degassed to $10^{-4}$ Torr at $370 \mathrm{~K}$ for three hours. By this treatment the oxygen adsorbed on the sample polymer had been sufficiently desorbed. When this predesorbed sample was used, the spectrum identical to Figure 6 was observed. However, no back reaction by the heat-treatment was observed for this sample.

\section{DISCUSSION}

In the experiments presented above comparison could be made between the various behaviors of the mechano-radicals and those of the $\gamma$ radicals. Because of the milling the sample flakes became finer and the total area of the surfaces of the finer flakes was increased after the milling. The increased surface area caused by fracture may play a role in the experiments: for example, on the reaction of the mechanoradicals with oxygen. The effects of the increased surface were checked and the obtained results were qualitatively similar to those described above. Moreover, it is impossible to separate the formation of the mechano-radicals from the increase of surface area in the milling 
experiments like ours, for the milling produces simultaneously both the mechano-radicals and the fresh surfaces. For the purpose of obtaining a qualitative understanding of the behaviors of the mechanical radicals in such a situation, the direct comparison of the non-fractured sample with the fractured one is useful and meaningful for the following discussion.

As mentioned in the previous section the greatly enhanced first stage in the isothermal decay of the peroxy mechano-radical, as shown in Figure 2, is interpreted as showing that $c a$. 90\% of the peroxy mechano-radicals are trapped in the amorphous region of the solid polypropylene. This interpretation is supported by the results obtained in the experiments of the temperature variation of the ESR line-shape of the peroxy mechano-radicals, because these experiments indicate that the peroxy mechanoradicals are trapped in a region where both the mobile molecular motions and the rapid decay are permitted at $40^{\circ} \mathrm{C}$, at which the molecular motion in the crystalline region is not so activated. ${ }^{22}$ In clear contrast with the behavior of the mechano-radicals, only 50 percent of the $\gamma$-radicals becay in the first stage in the isothermal decay (Figure 2) and their molecular motions at $40^{\circ} \mathrm{C}$ are not so mobile for the averaging (Figure 3-C).

One of the characteristic features of the mechano-radicals is their high reactivity with oxygen molecules. ${ }^{9}$ A hundred percent conversion to the peroxy radicals was obtained for the mechano-radicals but never for the $\gamma$-radicals. The species of PP mechano-radicals are different from the $\gamma$-radicals: the former are scission radi-

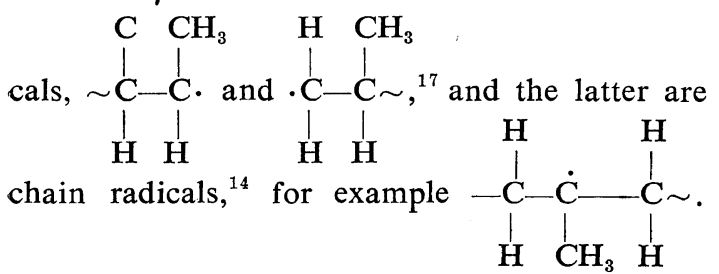

Thus, one might attribute the difference in the reactivity with oxygen to the difference in the species. However, the accessibility of oxygen molecules to the trapped radicals is more important for forming peroxy radicals, since a reaction in a solid phase is reasonably assumed to be determined by diffusion. ${ }^{11}$ Based on this assumption the high reactivity of the mechanoradicals with oxygen leads one to conclude that the mechano-radicals are trapped in the regions to which oxygen molecules are readily accessible, viz. surfaces. This conclusion is reconfirmed by the two cxperiments, the reactivity with MMA monomers and the photo-conversion. MMA monomer, which is too bulky to penetrate into the PP crystallites, scavenges rapidly all of the mechano-radicals, while doing only a few of the $\gamma$-radicals. This result demonstrates trapping of the mechano-radicals on the surfaces. In the photo-conversion experiments no back reaction was observed for the sample, in which oxygen had been carefully desorbed at higher temperatures. The reaction to the peroxy radicals by annealing is caused by the reaction of the radicals with the sorbed oxygen, and this reaction is repeated until the sorbed oxygens have been completely consumed. The above line of thought indicates clearly that the mechanoradicals are trapped on the surfaces, where the sorbed oxygen readily reacts with the radicals. The three independent experiments, the high reactivities with oxygen as well as with MMA monomers and the photo-conversion experiment, lead one to believe that the mechano-radicals are trapped on the surfaces. Since the surfaces are in a disordered or amorphous state, the mechano-radicals trapped in the surface layers are more mobile than in the crystalline state. Thus, trapping of the mechano-radicals is consistent with the conclusion that the mechanoradicals are more mobile than the $\gamma$-radicals at the temperatures studied. The small number of radical conversions induced by MMA, as shown in Figure 5, means that the $\gamma$-radicals are mostly trapped in the crystallite, into which bulky MMA monomers cannot penetrate. This is in accord with the conclusion derived from the isothermal decay behavior that roughly fifty percent in the first stage are in the amorphous region, but the radicals decaying with a very slow rate are trapped in the crystalline part. The above conclusion agrees quite well with the generally accepted picture that $\gamma$-irradiation produces the radicals homogeneously all over the sample and the probability to trap the radicals in the crystalline part is proportional to the crystallinity of the sample. Since the crystal. 

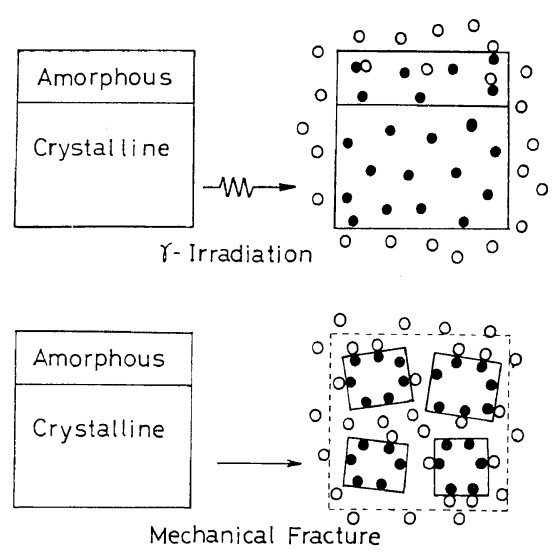

Figure 8. Schematic pictures of the trapping sites of both the mechano-radicals and the $\gamma$-radicals in a crystalline polymer: $O$, free radical; $\bigcirc$, adsorbed reactant.

linity of the sample used was nearly $70 \%$, it is understandable that the greater majority of the radicals formed by irradiation are trapped in the crystalline part. The arguments presented lead one to conclude that more than $90 \%$ of the mechano-radicals are trapped in the amorphous region, in spite of the fact that the amorphous fraction of the sample is less than 30 percent. This increase of the amorphous fraction was observed merely in the mechanical fracture and is explained by the creation of the fresh surfaces resulting from the fracture of the sample.

The various results described above convergently indicate that the PP mechano-radicals are formed and trapped on the fresh surfaces produced by the fracture. Comparison between the trapping sites of the mechano-radicals and the $\gamma$-radicals is schematically represented in Figure 8. This conclusion obtained for polypropylene may be extended more or less to other crystalline polymers. However, it is worth mentioning that there are experimental results which suggest that the mechano-radical migrates into the matrix in the case of amorphous cispolybutadiene. ${ }^{23}$

\section{REFERENCES}

1. P. Yu. Butyagin, I. V. Kolbanev, and V. A. Radtsig, Sov, Phys. Solid State, 5, 1642 (1964).
2. T. Kawashima, N. Nakamura, S. Shimada, H. Kashiwabara, and J. Sohma, Rep. Prog. Polym. Phys. Jpn., 12, 469 (1964).

3. S. N. Zhurkov and E. E. Tomashevsky in "Physical Basis of Yield and Fracture," A.C. Stickland, Ed., Inst. Phys. \& Phys. Soc. Conference Series No. 1, London, 1966, p 200.

4. A. Peterlin, J. Polym. Sci. Part A-2, 7, 1151 (1969).

5. H. H. Kausch, J. Macromol. Sci. Chem. C4, (2), 243 (1970).

6. K. L. DeVries, J. Polym. Sci., Part C, 32, 325 (1971).

7. T. Kawashima, S. Shimada, H. Kashiwabara, and J. Sohma, Polym. J., 5, 135 (1973).

8. M. Sakaguchi and J. Sohma, J. Polym. Sci., 13, 1233 (1975).

9. J. Sohma and M. Sakaguchi, Adv. Polym. Sci., 20, 111 (1976).

10. S. Shimada, H. Kashiwabara, and J. Sohma, J. Polym. Sci., Part A-2, 8, 1291 (1970).

11. H. Kashiwabara, S. Shimada, and J. Sohma, "ESR Applications to Polymer Research", Nobel Symposium 22, P. Kinell \& B. Rånby Ed., 1973, p 275 and Discussion p 288.

12. T. Nagamura, N. Kusumoto, and M. Takayanagi, J. Polym. Sci. Polym. Phys. Ed., 11, 2375 (1973).

13. H. Fischer and K. H. Hellwege, J. Polym. Sci., 56, 33 (1962).

14. H. Yoshida and B. Rånby, Acta Chem. Scand., 19, 72 (1965).

15. K. Tsuji, Adv. Polym. Sci., 12, 132 (1973).

16. Y. Hama, T. Ooi, M. Shiotsubo, and K. Shinohara, Polymer, 15, 787 (1974).

17. M. Sakaguchi, H. Yamakawa, and J. Sohma, Polym. Letters, 12, 193 (1974).

18. H. Fischer, K. H. Hellwege, and P. Neudörfl, J. Polym. Sci. Part A, 1, 2109 (1963).

19. B. Eda and M. Iwasaki, Polym. Letters, 7, 91 (1969).

20. S. Moriuchi, M. Nakamura, S. Shimada, H. Kashiwabara, and J. Sohma, Polymer, 11, 630 (1974).

21. R. J. Abraham, H. K. Melville, D. H. Ovenall, and D. H. Whiffen, Trans. Faraday Soc., 54, 1133 (1958).

22. N. G. McCrum, B. E. Read, and G. Williams, "Anelastic Dielectric Effects in Polymer Solids, John Wiley, New York, N.Y., 1976, Section 10.2 .

23. M. Yamamoto, M. Sakaguchi, M. Shiotani, and J. Sohma, Rep. Prog. Polym. Phys. Jpn., 16, 549 (1973). 